\title{
Towards Machine Learning Based Analysis of Quality of User Experience (QoUE)
}

\author{
Cosmas Ifeanyi Nwakanma, Md. Sajjad Hossain, Jae-Min Lee, and Dong-Seong Kim
}

\begin{abstract}
Industries use various platforms to receive feedback from users of their products. In this paper, there is an overview of the potentials of using natural language processing system (NLP) in classifying the quality of user experience. The user experience is captured using google form. To test the efficacy of the platform, sentiments of users were analysed using hotels.ng as the source of data. The natural processing of electronic word of mouth (e-WOM) can be applied to any feedback platforms to classify and predict customers' sentiments and provide a veritable opportunity for companies to capture the quality of users' experiences and improve service delivery. The feature or sentiments extraction was done using opinion mining and data cleaning tools on heterogeneous data sources to judge the decision-making process of users. Using charts and correlations, with an average performance level of the willingness to recommend and degree of review helpfulness, the platform showed that the Quality of User Experience (QoUE) of the customers are 7.31 and 7.03 respectively. Finally, an improved logistic regression classifier was developed to test, train and classify the user experiences. Comparing the improved logistic regression classifier with standard logistic regression classifier shows that the training accuracy of the proposed improved logistic regression gave $97.67 \%$ as against the standard logistic regression which had accuracy of $86.01 \%$
\end{abstract}

Index Terms-Machine learning, prediction, QoUE, users opinion mining, web crawling.

\section{INTRODUCTION}

It is considered that the expected launch of $5 \mathrm{G}$ and growth beyond $5 \mathrm{G}(\mathrm{B} 5 \mathrm{G})$, organizations are going to experience sophistication in web 2.0 and online democracy. Competition among various online shopping and booking platforms will be driven by the ability to capture, analyse and use customers' experiences to the organization's advantage. Examples of popular platforms where user experiences are captured and sentiments analysed include but not limited to Gmarket, booking.com, TripAdvisor, Facebook, hotels.ng, and such other organizations as Amazon and Google. These options

Manuscript received December 6, 2019; revised July 2, 2020. This work was supported by Priority Research Centers Program through the National Research Foundation of Korea (NRF) funded by the Ministry of Education, Science and Technology(2018R1A6A1A03024003). It is also supported by National Research Foundation of Korea (NRF) grant funded by Korea government (MSIT)(2019R1F1A1064055).

Cosmas Ifeanyi Nwakanma, Md. Sajjad Hossain, Jae-Min Lee, and Dong-Seong Kim are with the Networked Systems Laboratory, IT Convergence Engineering, School of Electronics Engineering, Kumoh National Institute of Technology, Gumi, Gyeongbuk 39177, South Korea; Cosmas Ifeanyi Nwakanma is also with the Department of Information Technology, School of Information and Communication Technology, Federal University of Technology, Owerri, Nigeria (Corresponding author: Dong-Seong Kim; e-mail: dskim@kumoh.ac.kr). available to the customer and low switching cost it affords is provided by the current trends in advancement of technology as well as the social media [1]-[3].

The primary motive for the sentiment analysis of user experience is to gain insight into how customers feel and respond to products and services. This work is intended to recommend possibilities of ensuring that the needs of customers are met, and their expectations exceeded in the future. This is considered important given that customer satisfaction is critical to the success of all businesses. Besides quality of experience, other researchers have also used sentiment analysis, otherwise known as emotion analysis, to detect speech, mood or language pattern [2]. In another related work, authors designed a framework, which can detect hate speech on Facebook due to the need to uncover the real intentions of Facebook users [4].

Similarly, machine learning models for analysing and predicting YouTube users' experiences, especially those using smartphones have been developed recently [5]. These and many other works, demonstrated the rising interest in measurement and monitoring quality of user experiences (QoUE) in recent times [6]. However, the challenge lies in capturing accurate and complete picture of the customer experience, considering that reviews and comments are now real time, just as data increase currently requires automation and machine learning. In this paper, the effect of online reviews on customers using hotels.ng as a case study was analyzed. The contributions of this paper include:

1) An overview of the evolving role of machine learning in capturing and analyzing quality of user experience using hotel.ng website as a case study.

2) A measure of the effect of customers' reviews on new customers, analyzing positive and negative feedback.

3) A review of challenges and open issues to the design and implementation of machine learning based analysis of sentiments derived in varied contexts from online platforms.

The rest of the paper is arranged as follows: in Section II, there is a brief background to three concepts - A. machine learning, B. quality of user experience and C. electronic word of mouth (e-WOM). Section III focuses on the overall system model. Section IV details the performance evaluation, while Section V concludes the paper.

\section{QUALITY OF USER EXPERIENCE AND MACHINE LEARNING}

\section{A. Machine Learning}

Machine learning has provided a veritable opportunity to 
monitor the moods and level of satisfaction of users of products and services in all areas such as industry, civil, military and social media [2]. It is therefore not surprising that in a bid to receive the best services, potential customers visit the review section of the platforms, in order to make informed decisions based on the experiences and opinions of past customers regarding various brands as can be seen in Fig. 1.

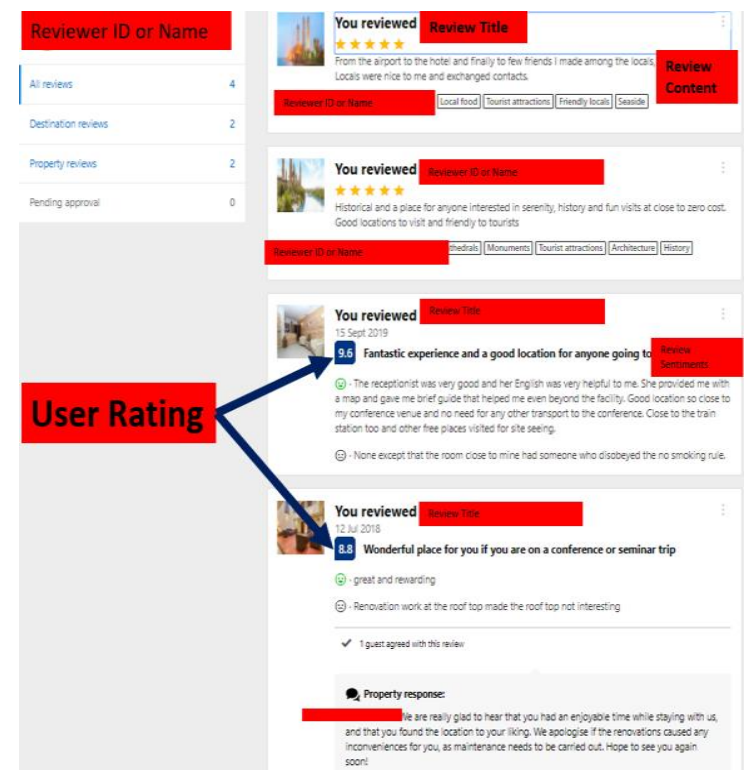

Fig. 1. QoUE sample of a typical platform [7].

\section{B. Quality of User Experience}

Machine learning is applied to the modeling of quality of experience (QoE), quality of service (QoS) and quality of user experience (QoUE) in networking for instance by using a set of indicators to represent the state and perception of users over a network [8]. This enables an automatic extraction and prediction of QoE or QoUE as the case may be. The researchers here argue that there is a need to guarantee reliable prediction, guided learning rate and careful design of such machine learning platform [8], [9].

As shown in Fig. 1, the features of the reviews used in this research work include:

- Review title: This is the header of the review. It is usually a short phrase or word that the guest uses to summarize his impression of the industrial product.

- Review content: This is the main area where the reviewer expresses his opinion in detail, about the industrial product.

- User rating: The rating here is a quantitative data. It has a possible value between 1-10; with 1 indicating strongly negative and 10 indicating strongly positive.

- User ID: This is the identity of the reviewer. Although the reviewers usually have the option of being anonymous, having User ID, lends the review a higher validity.

To manage and organize these diverse features better, machine learning tools are now employed in online reviews. They serve as credible source of past user perception and quality of experience to guide new or prospective users [10].
In some cases, reviews are presented in summarized formats to make it easier for the new visitors to quickly access what has been overtly said about the brand. Through these reviews, customers have quick and easy access to an unprecedented amount of user-generated product information which helps them choose the most appropriate product, according to their idiosyncratic preferences based on other customers' experiences [10]. The experiences and opinions of other customers can provide information about the quality and value of each product or service and hence reduce customers' risk in making choices. It also complements other forms of business to customer communication [11].

\section{C. $e-W O M$}

Online review, also called electronic word of mouth (e-WOM), has an impact on customer attitudes and consequently on booking or purchase intentions [12]. The influence of online review and its affects on the profitability of companies is repeatedly discussed in [13]. In the hospitality and tourism sector for example, online reviews influence prospective hoteliers and tourists as they make booking decisions. Studies on the state of social media [14], shows that $70 \%$ of respondents indicated they trust online consumer reviews. In [15], the relevance of the knowledge of quality of user experience, as captured in online reviews was emphasised as they have been shown to be of more impact than the traditional referrals.

However, of major concern is the level of correctness of online reviews since most reviewers tend to maintain an extreme positive and negative stance, based on their experiences [16]. All the same, this does not remove the impact of e-WOM on future purchasing decisions of users. Hence, prospective users rely more on the use of numerical ratings which are easy to process [17].

\section{SYSTEM MODEL}

\section{A. Overall Analytics Procedure}

Several steps or processes are involved in predictive analysis aimed at producing a model used to predict possible outcomes of a given instance. The processes are shown in Fig. 2. Under the modelling and pattern mining, the Mean Opinion Score (MOS) model for over the top content is given as Eqn.(1) where $x$ denotes the number of product purchase and $t$ is the time since last purchase and $g$ represents the memory parameter sometimes set at a typical value as 0.14 [18].

$$
\operatorname{MOS}=e^{-\frac{x}{5}+1.5-g \sqrt[e]{t}}
$$
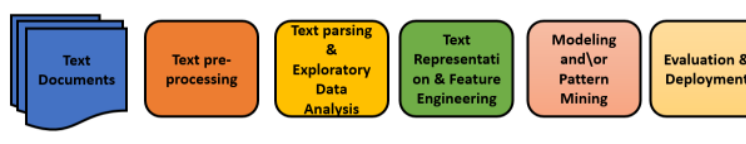

Fig. 2. Text analysis procedure.

\section{B. Predictive Model}

Predictive models use many several techniques in 
preparing data for modeling, estimation, validating, scoring data, or related mining activities which leverage data mining, statistics, modeling, machine learning, and artificial intelligence to analyze current data and to make predictions about the future. The aim here is to ascertain what has happened, as to provide the best estimation of what will happen in the future [19]. This study shows how patterns found in historical and transactional data can be translated into algorithms and used to identify risks and opportunities for the future. When these algorithms are tested over time, especially in multiple scenarios, the model makes predictions with new data introduced into the system. Fig. 3 shows the outcome of the predictive model, where the model can make predictions of the future from the information feed as input just like an expert system.

This paper compared improved logistic regression classifier with standard logistic regression. The evaluation of the model accuracy was done using the standard Equation (2) adapted from [20]. In Equation (2), $\varepsilon$ is the number of true positives while $\alpha$ is the number of false positives. The precision is a measure of the ability of the classifier to not label a sample as positive if it is negative. Another measure is "recall" which is a measure of the classifier's ability to find all the positive samples. Recall is given as Equation (3) where $\beta$ is the number of false negatives. The third measurement criteria are the "F-beta" score which lies between the values of 0 (worst case) and 1 (best case).

$$
\begin{aligned}
& \varepsilon /(\varepsilon+\alpha) \\
& \varepsilon /(\varepsilon+\beta)
\end{aligned}
$$

(1)

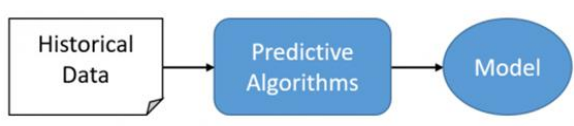

2

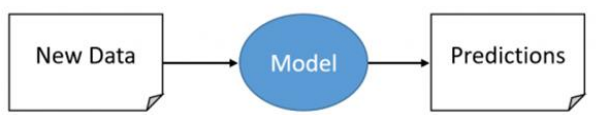

Fig. 3. Predictive model.

The use of this model helps to avoid prediction challenges such as equipment failure or depleted stock, or capitalizing on opportunities to market products to customers, like targeting people in happy or dejected moods after a sporting event [21].

\section{Sentiment Analysis}

Sentiment analysis is the automated process of classifying online text data as positive, neutral or negative, giving businesses the opportunity to gain a deeper insight into customers' assessment of their products, brand or services. Text mining (also referred to as text analytics) is an artificial intelligence (AI) technology that uses natural language processing (NLP) in transforming the free (unstructured) text in documents and databases into normalized, structured data suitable for analysis or to drive machine learning (ML) algorithms. Arguably, NLP becomes a machine learning tool once its parameters are automatically learned from data.
This involves an automated detection of users' assessment of their products and services as they are posted in online platforms for the purpose of feedback. Such platforms could be websites of the companies, social media handles like twitter, or any booking or even online shopping platforms such as Gmarket. The essence is to identify, analyse and codify the opinions of users based on user experience and satisfaction. With the growth of web 2.0, opinions can be expressed even without the use of texts, as the use of images and emojis now dominate the social media. Thus, extracting even smiley and symbols can also be a source of feedback to companies and organizations. This development has placed the study of opinion mining and sentiment analysis on the burner of both academics and industry.

Manufacturers as well as service companies now rely on the power of machine learning for the aggregation of feedback via sentiment analysis. With this, an unstructured response could be structured into a meaningful format that provides relevant reviews to prospective users. The new format is then readily available as a means of branding, customer service support and feedback for re-work in the case of manufacturing companies who rely on feedback from users of their products to gain a competitive edge.

Experts believe that customers are most likely to spend $31 \%$ more on businesses with excellent reviews [22]. From the analysis of respondents' viewpoints, about $72 \%$ said positive reviews make them trust local businesses, $72 \%$ will take action after reading positive reviews and $86 \%$ will hesitate to purchase from a business with negative online review. With the conclusions drawn from several studies, online reviews have become an important conversation for hospitality and tourism industry. This is so because people who require quality comfort and relaxation service can only learn about them through the said online platforms.

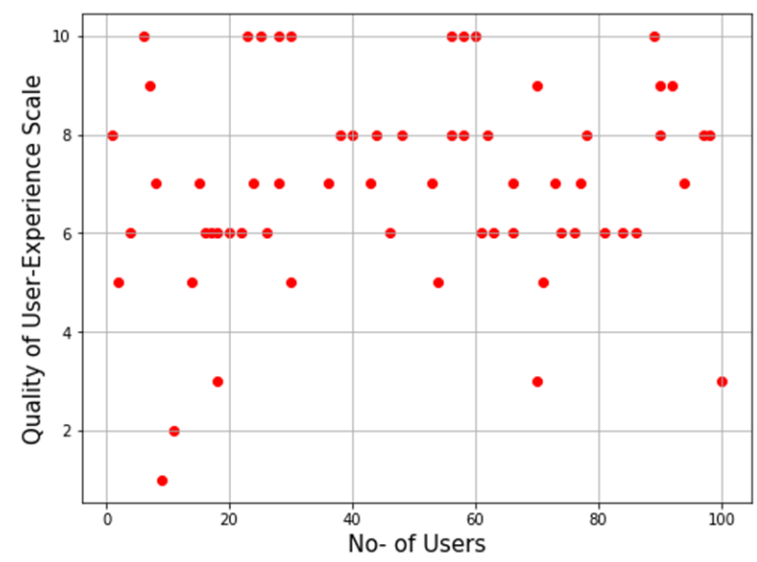

Fig. 4. Spread of sentiments of 3000 users.

\section{Research Design}

In this study, hotels.ng was used as the main platform to aggregate the comments. Hotels.ng was selected due to data availability to the authors. The platform is just like any other booking platform and as a ready source of online reviews, it is very relevant to this research. A questionnaire was developed for primary data collection and web scrapping was used to extract reviews for sentiment classification. The 
questionnaire bubble rating is shown as a 10-point scale and a decision rule was formulated to guide the grading of each rate: $1=$ "Extremely Terrible", $2=$ "Moderately Terrible", $3=$ "Terrible", 4= "Poor", 5 = "Moderate", 6 = "Good", 7= "Very Good", 8= "Super Good", 9= "Nearly Excellent" and $10=$ "Excellent". While the sentiment classification was graded into negative weighted, neutral weighted and positive weighted. Hypotheses were formulated to examine the relationship between features of a hotel and its importance to hotel users, online reviews and its impact on the brand. The spread of sentiments captured from three thousand respondents is shown in Fig. 4.

The dataset was collected using web crawling technique to extract data from Hotels.ng website. Web crawling involves implementing a software to surf the internet with the sole aim of mining relevant data or opinion [23]. This paper used a Java library called "Jsoup" to collect user reviews from hotels.ng. One challenge facing the use of Web scraping is privacy. In order to ensure that the privacy of their customer is preserved, some websites do not permit certain kinds of data mining. Web scraping however will continue to enjoy wide range of usage despite this legal obstacle due to its role as data mining tool which is useful for feedback and prediction.

A self-constructed questionnaire created with Google forms tools was used to gather data about how users make decisions on a product and how online reviews influence such decisions. The Google forms used were distributed by posting the link on WhatsApp between September 2018 and November 2018. The questionnaire was made up of seven (7) questions, with two questions being on linear scale, two (2) multiple choice questions and three (3) open questions for selection. Google form can be created by signing up to have a Google account, after which the email address can be used to access the form. The structure of the form and questions it contains will be determined by the creator. After the creation of the form, the URL can be sent out to the target respondents. The responses are then saved in the drive of the email address used in creating the form. These responses can also be exported to a spread sheet. In this paper, the spreadsheet was converted to a Comma Separated Values (CSV) file to reduce training time complexity associated with the use of excel. Fig 5 shows the time scaled representation of Fig. 4.

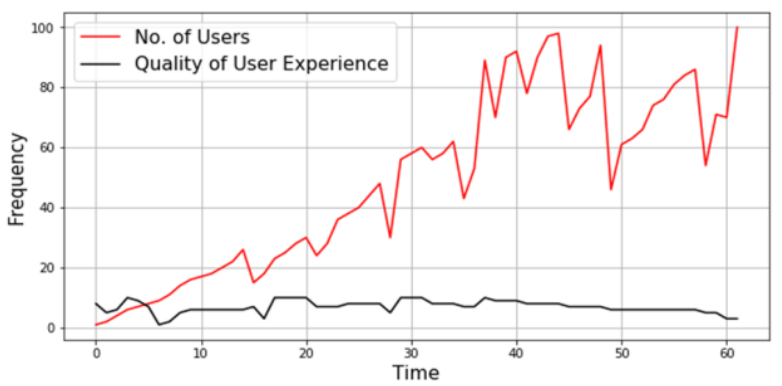

Fig. 5. Frequency plot of the sentiments over the time of data collection.

The population study for the online review consist of a community user of hotels.ng platform, while the respondents for the questionnaire consists of wide range of people available online at the time it was uploaded on the social. The population spans across all age ranges and both sexes who are in the community of hotels.ng users and internet users.

\section{PERformance EVAluation AND OPEN RESEARCH ISSUES}

\section{A. Performance Evaluation}

Upon the implementation of the predictive analytics tool, the test run showed the following results captured from 3000 persons who were asked to give their opinion using Google form as a means of response collection. Fig. 6 shows the willingness of customers to recommend a product after usage. This recommendation can influence other prospective users, while they dabble into online in search. In Fig. 6 and Fig. 7, the average willingness to recommend a product and degree of review helpfulness is 7.31 and 7.03 respectively. This figure on a scale of 0 to 10 validates the premise of this paper which states that sentiments or opinion of customers affect or influence future patronage of industrial products and services. To classify the QoUE, an improved logistic regression model developed, and its performance compared with conventional logistic regression. Fig. 8 shows the confusion matrix while Fig. 9 shows the classification into satisfied and dissatisfied users.

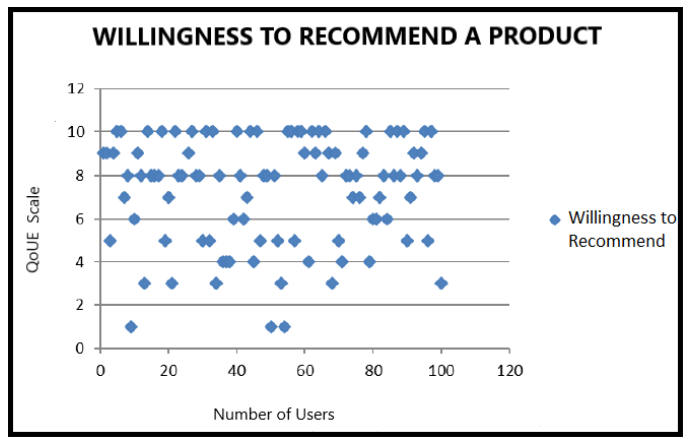

Fig. 6. Willingness to recommend a product.

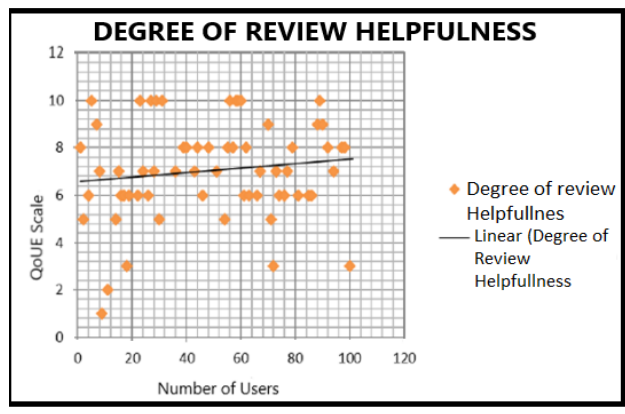

Fig. 7. Degree of review helpfulness.

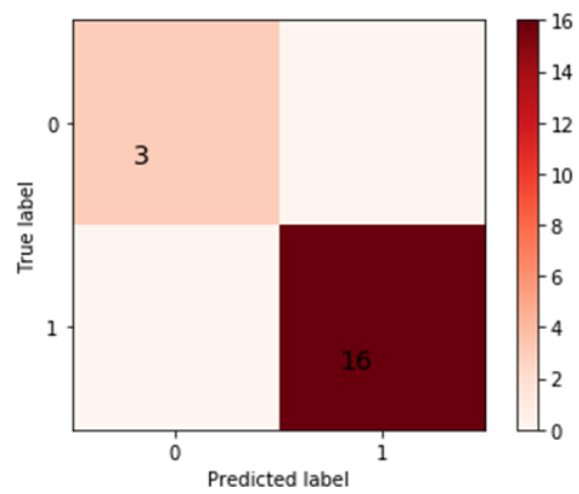

Fig. 8. Confusion matrix of improved logistic regression classifier. 


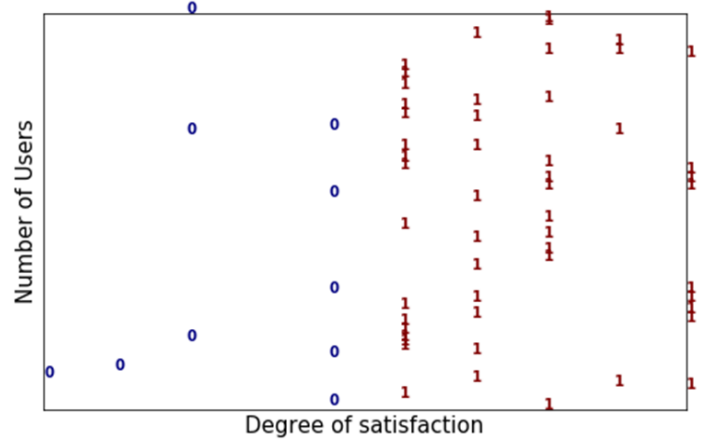

Fig. 9. Improved logistic regression classification of sentiments into dissatisfied (0) and satisfied (1).

1) Accuracy Score of Model: This paper compared the improved logistic regression model with conventional logistic regression. This was done using python 3.7 and Jupyter notebook. The Jupyter Notebook is an open-source web application that allows you to create and share documents that contain live code, equations, visualizations and narrative text. Uses include data cleaning and transformation, numerical simulation, statistical modeling, data visualization, machine learning, and much more. Table I and Table II shows that the improved logistic regression model performed better than the standard regression model during training and test. Training accuracy of the improved logistic regression gave $97.67 \%$ against the standard logistic regression which gave accuracy of $86.01 \%$. Similarly, the test accuracy of improved logistic regression model gave $100 \%$ against that of the standard logistic regression which was $94.74 \%$. In line with the conventional practice in most machine learning projects, $70 \%$ of the dataset was used for training, while $30 \%$ was used for testing [20]. The improved logistic regression classifier showed good performance judging by Table III as well as Fig. 10 where the loss is minimal as epoch is increased compared to the conventional logistic regression classifier.

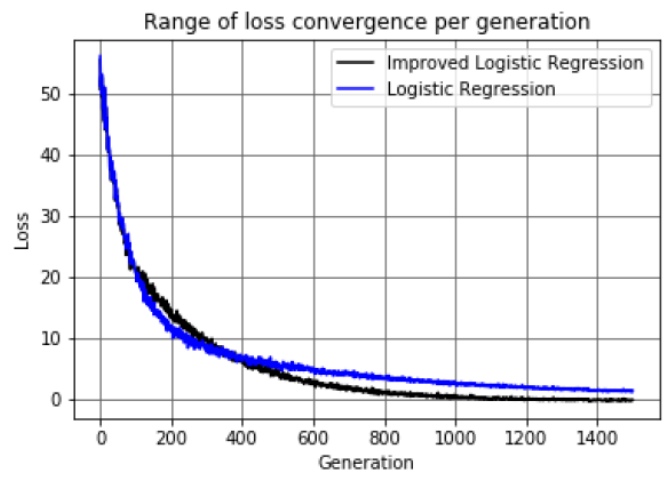

Fig. 10. Range of loss convergence comparing the performance of improved logistic regression classifier with the conventional logistic regression classifier.

TABLE I: ACCURACY SCORE OF TRAINING

\begin{tabular}{ll}
\hline \hline Algorithm & Accuracy \\
\hline Logistic Regression Classifier & $86.01 \%$ \\
Logistic Regression Classifier & $97.67 \%$ \\
\hline \hline
\end{tabular}

TABLE II: ACCURACY SCORE OF TESTS

\begin{tabular}{lc}
\hline \hline Algorithm & Accuracy \\
\hline Logistic Regression Classifier & $86.01 \%$ \\
Logistic Regression Classifier & $97.67 \%$ \\
\hline \hline
\end{tabular}

2) Classification Report: The computed precision, recall and F-beta score is as shown in Table III.

TABLE III: CLASSIFICATION REPORT OF IMPROVED LOGISTIC REGRESSION

\begin{tabular}{llll}
\hline \hline Channels & Precision & Recall & F-Beta Score \\
\hline Not Satisfied Users & 1 & 1 & 1.00 \\
Satisfied Users & 1 & 1 & 1.00 \\
\hline \hline
\end{tabular}

\section{B. Open Research Issues}

As interesting as the use of machine learning and artificial intelligence may be in the capturing of QoUE, three research issues are worthy of note. These research issues are: Extreme review of users, Data set access and dynamic update by some review platforms, Legal and ethical issues of web crawling and Bidirectional Encoder Representations from Transformers (BERT).

1) Extreme review of users: Machine learning models are as good as the quality of data provided. This has raised concern over the level of reliability of reviews provided by users. Since some reviewers can be suspected to review at extreme, their review may not be reliable and devoid of sentiments or bias. There is, therefore, the need to develop ways to normalise the effect of outliers on prediction. This problem was a challenge to [24], in their design of a recommender system that serves as a source of review of products for potential users. In solving the problem, they relied on the users' rating instead of written reviews alone. More research and approach to identify extreme reviews and isolate them before translating them to ratings is expected

2) Data set access and dynamic update by some review platforms: As evident in this work, the source of data is an online platform and one common feature to most online review platforms is the continuous update of their website. In fact, Facebook now sieves some words posted by users using various rules and algorithms. This poses a restriction and re-usability of data. However, the challenge of limited data can be solved by the use of hybrid deep learning frameworks as suggested by [25]. It is expected by researchers that hybrid model frameworks will dominate future machine learning designs. Possibly too, there would be a comparison of various hybrid models for specific target systems.

3) Legal and ethical issues of web crawling: The concept or practice of web crawling involves using software or any means to extract information from the internet and in most cases, without official permission of the websites or platform owners. This has generated a lot of legal and ethical concerns with various people holding divergent opinion. Web crawling also known as web harvesting can be seen as a form of data mining, but the legal issues remain a research direction for both the industry and academia. However, despite these concerns, web crawling is gaining relevance and usage. This calls for more research on how to set up best practices to reach a tradeoff between its importance and potential threat.

4) Bidirectional Encoder Representations from Transformers (BERT): Recent works now employ 
Google's BERT for predicting experiences and usage in clinical dialogues [26], [27]. Google's BERT is a state-of the-art model released. It is commanding wide usage by researchers in the field of natural language processing. The open issues for researchers centre on the effective usage and adaptation to various scenarios. For instance, in a recent work, there was a reported challenge faced by their researchers on how to use the BERT [26]. They compared the performance of BERT to a rule-based system and found that BERT did not perform optimally as claimed by inventors. However, they quickly accepted that their findings may be due to inability to test all possible ways to use BERT [26]. It is therefore a research direction to see more BERT-based systems emerge in the coming future. Similarly, another research trained their BERT model and compared its performance with that of Google's pre-trained models [27]. The result showed that their model outperformed that of Google's BERT in predicting personal experience using medication-related tweets as a case study [27].

\section{CONCLUSION}

This paper presents an overview of machine learning particularly natural language processing tools for the analysis of users satisfaction measured by QoUE metrics. The results showed that the extracted sentiments using the web scraping technique corroborates the opinion of respondents in the questionnaire. This showed the potential and capability of machine learning in analysing and predicting large database of sentiments or reviews. This approach can be applied to industrial scenarios where quality control units can use QoUE to appraise the perception of users of their products and services. Finally, an improved logistic regression classifier was developed to test, train and classify the user experiences. Comparing the improved logistics regression classifier with standard logistic regression classifier shows that the training accuracy of the improved logistic regression gave $97.67 \%$ as against the standard logistic regression which gave accuracy of $86.01 \%$.

\section{CONFLICT OF INTEREST}

The authors declare no conflict of interest.

\section{AUTHOR CONTRIBUTIONS}

Cosmas Ifeanyi Nwakanma proposed the paper idea, collected data and wrote substantial part of the paper as well as the MATLAB analysis and python codes for the logistic regression. Md Sajjad Hossain assisted in the improvement of the paper quality and helped in completing paper writing. Jae-Min Lee and Dong-Seong provided the supervision leading to the comprehensive enhancement of paper after each review stage. Dong-Seong Kim is the Professor in charge of Networked System Laboratory responsible for the research funding. All authors had approved the final version.

\section{ACKNOWLEDGMENT}

Authors are grateful to Mr Ugochukwu Ejike Akpudo of the Defence Reliability Laboratory, Kumoh National Institute of Technology, Gumi, South Korea for assisting in the python codes and technical support towards the revision of this paper. Authors are also grateful to Dr. Uchenna David Uwakwe, Directorate of General Studies, Federal University of Technology Owerri, Nigeria for English correction service.

\section{REFERENCES}

[1] P. Kachamas, S. Akkaradamrongrat, S. Sinthupinyo, and A. Chandrachai, "Application of artificial intelligent in the prediction of consumer behavior from Facebook posts analysis," International Journal of Machine Learning and Computing, vol. 9, no. 1, pp. 91-97, February 2019.

[2] M. C. Lee, J. Kim, and D. S. Kim, "Multimedia content recommendation in social networks using mood tags and synonyms," Multimedia Systems, August 2019.

[3] C. I. Nwakanma, A. C. Ogbonna, C. Etus, E. U. Nwifor, J. E. Onyebuchi, and E. C. Ugwueke, "Predictive analytics of customer sentiments towards Nigerian hospitality industry: Case study approach," in Proc. $3^{\text {rd }}$ International Conference on Intelligent Computing and Emerging Technologies (ICET 2019), Babcock University Ogun State, Nigeria, pp. 60-68.

[4] A. Rodríguez, C. Argueta, and Y. Chen, "Automatic detection of hate speech on facebook using sentiment and emotion analysis," in Proc. 2019 International Conference on Artificial Intelligence in Information and Communication (ICAIIC 2019), Okinawa, Japan, pp. 169-174.

[5] S. Wassermann, N. Wehner, and P. Casas, "Machine learning models for YouTube QoE and user engagement prediction in smartphones," in Proc. 2019 Workshop on AI in Networks (WAIN), Toulouse, France, 2018.

[6] M. Seufert, S. Wassermann, and P. Casas, "Considering user behavior in the quality of experience cycle: Towards proactive QoE-aware traffic management," IEEE Communications Letters, vol. 23, no. 7, pp. 1145-1148, July 2019.

[7] B.V. Amsterdam in the Netherlands 1996-2020. [Online]. Available: http://www.Booking.com.

[8] S. Aroussi and A. Mellouk, "Survey on machine learning-based QoE-QoS correlation models," in Proc. 2014 International Conference on Computing, Management and Telecommunications (ComManTel), Da Nang, 2014, pp. 200-204.

[9] W. C. Lee and D. K. Yoon, "A study on facial expression and first impression through machine learning," in Proc. 2019 International Conference on Artificial Intelligence in Information and Communication (ICAIIC 2019), Okinawa, Japan, pp. 298-301.

[10] S. K. Daniel, R. Jochen, M. Natter, and D. Clapper, "Decomposing the effects of online customer reviews on brand, price, and product attributes," International Journal of Research in Marketing, vol. 33, No 1, pp. 11-26, March 2016.

[11] C. Efthymios, and I. H. Nina, "Impact of online product reviews on purchasing decisions," in Proc. the 12th International Conference on Web Information Systems and Technologies (WEBIST 2016), LDA: SCITEPRESS - Science and Technology Publications, 2016, pp. 271-178.

[12] R. Ladhari, and M. Michaud, "eWOM effects on hotel booking intentions, attitudes, trust, and website perceptions," in Proc. International Journal of Hospitality Management, April 2015, vol. 46, pp. 36-45.

[13] J. Chevalier and D. Mayzlin, "The effect of word of mouth on sales: Online book reviews," Journal of Marketing Research, vol. 43, no. 3, pp. 345-354, 2006.

[14] Nielsen, "State of the media," The Social Medeia Report, 2012.

[15] C. M. Edward, M. Ewa, and F. B. Stefan, "The effect of online customer reviews' characteristics on sales," Advances in Advertising Research, 2018.

[16] B. Katerina, A. Bilgihan, C. Cihan, and O. Fevzi, "Understanding satisfied and dissatisfied hotel customers: Text mining of online hotel reviews," Journal of Hospitality Marketing and Management, vol. 25, no. 1, pp. 1-24, 2015.

[17] X. Zhao, L. Wang, X. Guo, and R. Law, "The influence of online reviews to online hotel booking intentions," International Journal of Contemporary Hospitality Management, vol. 27, no. 6, pp. 1343-1364, 2015.

[18] U. Bulkan and T. Dagiukals, "Predicting quality of experience for online video service provisioning," T. Multimed Tools Appl, vol. 78, no 13, pp. 18787-18811, 2019.

[19] E. Siegel, "The power to predict who will click, buy, lie or die," Predictive Analytics, 2013. 
[20] F. Khennou, C. Fahim, H. Chaoui, and N. E. H. Chaoui, "A machine learning approach: Using predictive analytics to identify and analyze high risks patients with heart disease," International Journal of Machine Learning and Computing, vol. 9, no. 6, pp. 762-767, December 2019.

[21] Reportlinker, "Predictive analytics - global market outlook (2017-2030)," PR Newswire, 2018.

[22] K. Saleh, "The importance of online customer reviews [infographic]," Invespro, September 2017.

[23] P. K. Duvvur, Predicting Hotel Rating Based on User Reviews, 2016.

[24] N. A. Osman, S. A. M. Noah, and M. Darwich, "Contextual sentiments based recommender system to provide recommendation in the electronic products domain," International Journal of Machine Learning and Computing, vol. 9, no. 4, pp. 425-431, 2019.

[25] V. Gavrishchaka, Z. Yang, R. Miao, and O. Senyukova, "Advantages of hybrid deep learning frameworks in applications with limited data," International Journal of Machine Learning and Computing, vol. 8, no. 6, pp. 549-558, 2018.

[26] C. Lin, C. Huang, and C. Wu, "Using BERT to process Chinese Ellipsis and coreference in clinic dialogues," in Proc. 2019 IEEE 20th International Conference on Information Reuse and Integration for Data Science (IRI), Los Angeles, CA, USA, 2019, pp. 414-418.

[27] K. Jiang, T. Chen, R. A. Calix, and G. R. Bernard, "Prediction of personal experience tweets of medication use via contextual word representations*," in Proc. 2019 41st Annual International Conference of the IEEE Engineering in Medicine and Biology Society (EMBC), Berlin, Germany, 2019, pp. 6093-6096.

Copyright $(\odot 2020$ by the authors. This is an open access article distributed under the Creative Commons Attribution License which permits unrestricted use, distribution, and reproduction in any medium, provided the original work is properly cited (CC BY 4.0).

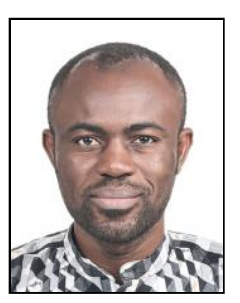

Cosmas Ifeanyi Nwakanma is currently a $\mathrm{PhD}$ student and full-time researcher at Networked System Laboratory, IT-Convergence Engineering in Kumoh National Institute of Technology Gumi, South Korea. $\mathrm{He}$ received his national diploma (distinction) in electrical/electronic engineering from Federal Polytechnic Nekede Imo State Nigeria in 1999. He later received his bachelor of engineering in communication engineering in 2004, master's in information technology in 2012 and master of business administration (MBA) in project management technology in 2016 all from the Federal University of Technology Owerri Imo State Nigeria, where he has put in ten years' lecturing and research experience. He was an intern with Asea Brown Boveri (ABB) Nigeria in 2003. As a member of IEEE, he has served as a volunteer reviewer to IEEE Access. His research interests are Reliability and Prediction in Real-Time Industrial Networked Systems using machine learning.

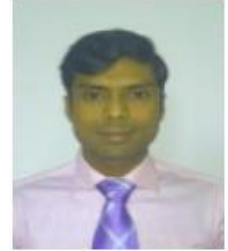

Md. Sajjad Hossain received his B.Sc in electronics and telecommunication engineering from Rajshah University of Engineering and Technology (RUET), in December 2016. He is currently a master's student and graduate research assistant with the Networked Systems Lab. (NSL), of the School of Electronics Engineering, Kumoh National Institute of Technology, Gumi, South Korea. His research area includes edge/fog computing and the industrial IoT (IIoT)

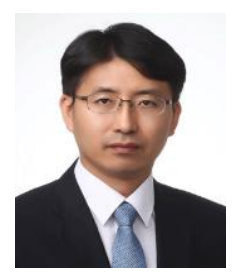

Jae-Min Lee received the $\mathrm{PhD}$ degree in electrical and computer engineering from Seoul National University Seoul, Korea in 2005. From 2005 to 2014, he was a senior engineer with Samsung Electronics, Suwon. From 2015 to 2016, he was a principal engineer in Samsung Electronics, Suwon, Korea. Since 2017, he has been an assistant professor with the School of Electronic Engineering and Department of IT Convergence Engineering, Kumoh National Institute of Technology, Gyeongbuk, Korea. He is a member of IEEE. His current research interests are industrial wireless control network, performance analysis of wireless networks and TRIZ

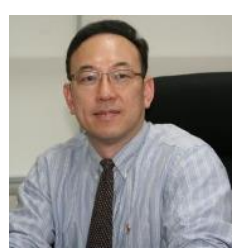

Dong-Seong Kim received his Ph.D. degree in electrical and computer engineering from the Seoul National University, Seoul, Korea, in 2003. From 1994 to 2003, he worked as a full-time researcher in ERC-ACI at Seoul National University, Seoul, Korea. From March 2003 to February 2005, he worked as a postdoctoral researcher at the Wireless Network Laboratory in the School of Electrical and Computer Engineering at Cornell University, NY. From 2007 to 2009, he was a visiting professor with the Department of Computer Science, University of California, Davis, CA. He is the director of KIT Convergence Research Institute and ICT Convergence Research Center (ITRC and NRF advanced research center program) supported by Korean government at Kumoh National Institute of Technology. He is currently the dean of Industry Academic Cooperation Foundation as well as the lead professor in charge of Networked Systems Laboratory, School of Electronic Engineering, Kumoh National Institute of Technology, South Korea. He is a senior member of IEEE/ACM, and the Executive Manager of the Korean Institute of Communications and Information Sciences. His current research interests are real-time IoT and smart platform, industrial wireless control network, networked embedded system. 